\section{International Scientific Journal Theoretical \& Applied Science}

Svetlana Valeryevna Fedorova Candidate of Technical Sciences, associate professor Irkutsk National Research Technical University, Russia

p-ISSN: 2308-4944 (print) e-ISSN: 2409-0085 (online) $\underline{\text { fsta@istu.irk.ru }}$

Year: $2015 \quad$ Issue: 09 Volume: 29

Published: $30.09 .2015 \quad$ http://T-Science.org

SECTION 12. Geology. Anthropology. Archeology.

\title{
MATHEMATICAL MODEL OF OPTIMIZATION OF PROCESS OF CRUSHING OF GLASS IN THE JET MILL
}

Abstract: Mathematical modeling of processes of crushing of aluminosilicates, namely I flew down, it is intended for studying of the principles of creation of computer models of optimization of process of crushing. These results will help to answer the specific questions containing in technology of receiving composite materials on the basis of mica and glass.

Key words: micag, lass, mathematical model, optimization.

Language: Russian

Citation: Fedorova SV (2015) MATHEMATICAL MODEL OF OPTIMIZATION OF PROCESS OF CRUSHING OF GLASS IN THE JET MILL. ISJ Theoretical \& Applied Science 09 (29): 89-92.

Soi: http://s-o-i.org/1.1/TAS-09-29-19 Doi: crossef http://dx.doi.org/10.15863/TAS.2015.09.29.19

\section{МАТЕМАТИЧЕСКАЯ МОДЕЛЬ ОПТИМИЗАЦИИ ПРОЦЕССА ИЗМЕЛЬЧЕНИЯ СТЕКЛА В СТРУЙНОЙ МЕЛЬНИЦЕ}

Аннотация: Математическое моделирование процессов измельчения алюмосиликатов, а именно стекла, предназначено для изучения принципов построения компьютерных моделей оптимизации процесса измельчения. Эти результаты помогут ответить на конкретные вопросы, содержашиеся в технологии получения композиционных материалов на основе слюды и стекла.

Ключевые слова: Слюда, стекла, математическая модель, оптимизация.

Теоретически точное описание процесса измельчения стекла требует учета широкого спектра факторов. Прежде всего, это затраты энергии на предельные упругие и пластические необратимые деформации, которые определяют характер модели.

Наряду с этими, имеются и другие затраты энергии: на трение, на образование агрегатов и т.д. В силу малой изученности последних, не теряя качественной адекватности модели, можно ограничиться рассмотрением предельных упругих деформаций.

С известной степенью приближения можно считать, что

$$
\mathrm{Dw}=\mathrm{K}-\mathrm{d} \varepsilon\left(1-\mathrm{S} / \mathrm{S}_{\mathrm{m}}\right),
$$

где $\mathrm{W}$ - плотность энергии, передаваемой частице. $\mathrm{S}$ удельная поверхность частицы, $\mathrm{S}_{\mathrm{m}}$ удельная поверхность предельно измельченного порошка, $\varepsilon$ -энергия, затраченная измельчителем, К - КПД измельчителя. В процессе измельчения удельная поверхность частиц $\mathrm{S}$ приближается к значению удельной поверхности $\mathrm{S}_{\mathrm{m}}$ измельченного порошка, с некоторым заданным размером частиц.

Интегрированием уравнения (1), подробно см. Г.С. Ходаков "Тонкое измельчение строительных материалов", и дельнейшими преобразованиями получаем уравнение степени измельчения

$$
\mathrm{S}=\mathrm{S}_{\mathrm{m}}\left(1-\mathrm{e}^{-\mathrm{K}_{2}} \mathrm{t}\right)
$$

где - $\kappa_{2}=\kappa_{1} ;$. N /V, N- мощность измельчителя, $\mathrm{V}$ - объем измельчаемого порошка, К - КПД ударного разрушения частицы, равный отношению энергии разрушения к общей величине кинетической энергии частицы перед ударом. Важнейшим содержанием этой модели является экспоненциальный характер асимптотического приближения к кондиционному значению $\mathrm{S}_{\mathrm{m}}$. Определенную специфику придает то, что $\mathrm{V}$ не является постоянным в течении процесса. Из (2) следует, что уменьшение V приводит к более быстрой сходимости $\mathrm{S}$ к $\mathrm{S}_{\mathrm{m}}$. 


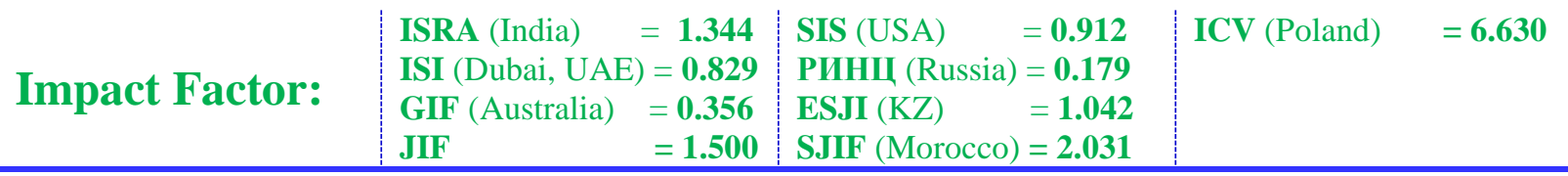

Качественная модель определяет подход к построению регрессии на основе экспериментальных данных, представленных ниже для фракции $0.1<\mathrm{S}_{\mathrm{m}}<0.2$.

Таблица 1

\begin{tabular}{|c|c|c|c|}
\hline Т,ч & 0,5 & 1 & 1,5 \\
\hline Выход, у & 0,412 & 0,609 & 0,89005 \\
\hline
\end{tabular}

Уравнение регрессии процесса измельчения стекла в струйной мельнице имеет вид:

$\mathrm{y}=1-\mathrm{at}^{2} * \mathrm{e}^{-\mathrm{bt}}$

(3)

Здесь у - оценка выходного параметра модели (процент выхода необходимой фракции.); экспоненциальная составляющая отражает качественное поведение модели, а квадратический член ее специфику; $\mathrm{t}$ - входной параметр (время). Применяя логарифмирование для (3), получим

$\operatorname{Ln}(1-\mathrm{y})=\mathrm{Lna}+2 \mathrm{Lnt}-\mathrm{bt}$.

Пусть $1-\mathrm{y}=\mathrm{x}, \mathrm{x}=\left(\mathrm{x}_{1}, \mathrm{x}_{2}, \mathrm{x}_{3}\right)=(0,588,0,391$, $0,10995), t=\left(t_{1} t_{2} t_{3}\right)=(0,5,1,1,5)$.
Для регрессии воспользуемся методом наименьших квадратов, находя постоянные a, b, из следующей системы нормальных уравнений:

$\{\Sigma \mathrm{Ln},=\mathrm{n} \mathrm{Ln} \mathrm{a}+2 \mathrm{~S} \operatorname{Ln} \mathrm{ti}+\mathrm{b}(\Sigma \mathrm{ti})$

$\left\{\Sigma(\mathrm{ti}\right.$ Ln xi $)=(\Sigma \mathrm{ti}) \operatorname{Ln} \mathrm{a}+2 \Sigma(\mathrm{t} \mathrm{Ln} \mathrm{ti})+\mathrm{b}\left(\Sigma \mathrm{ti}^{2}\right)$, $\left\{\Sigma(\right.$ Ln ti $\left.\mid * \operatorname{Ln} \mathrm{xi})=(\Sigma \mathrm{Ln} \mathrm{ti}) * \operatorname{Ln} \mathrm{a}+2 \Sigma \mathrm{Ln} \mathrm{Xi^{2 }}\right)+$ $\mathrm{b}\left(\sum \mathrm{ti} \operatorname{Ln} \mathrm{ti}\right)$,

где $\mathrm{n}=3$, количество испытаний. Подставляя известные значения у и $\mathrm{t}$ из таблицы, решаем систему. В результате, $\mathrm{a}=16.3173, \mathrm{~b}=3,8739$.

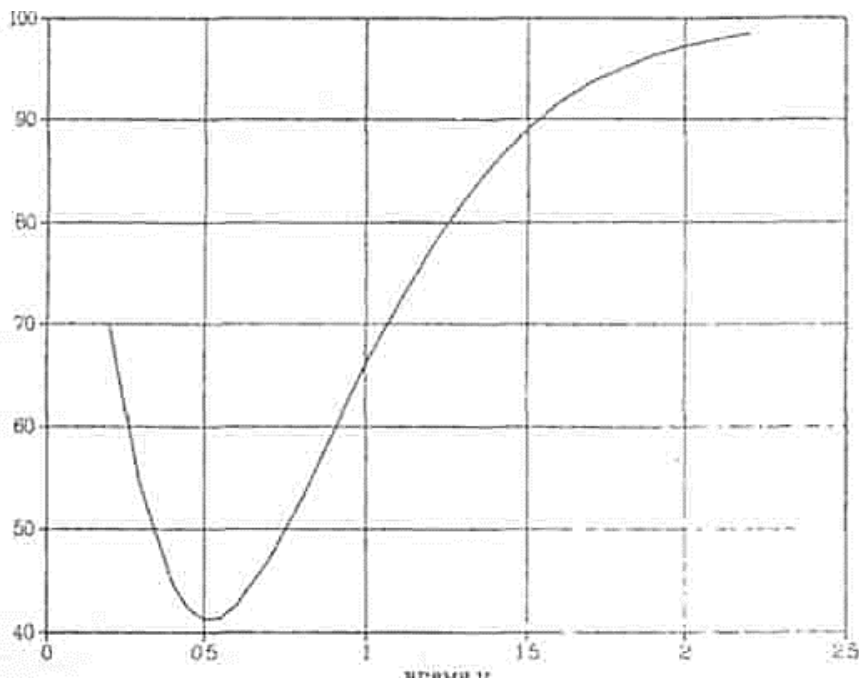

Рисунок 1 - Общий вид кривой, полученной в результате регрессионного анализа по опытным данным. 


\begin{tabular}{|c|c|c|c|c|c|c|}
\hline Impact Factor: & $\begin{array}{l}\text { ISRA (India) } \\
\text { ISI (Dubai, UAE } \\
\text { GIF (Australia) } \\
\text { JIF }\end{array}$ & $\begin{array}{l}=1.344 \\
=0.829 \\
=0.356 \\
=1.500\end{array}$ & $\begin{array}{l}\text { SIS (USA) } \\
\text { PИНЦ (Russia) } \\
\text { ESJI (KZ) } \\
\text { SJIF (Morocco) }\end{array}$ & $\begin{array}{l}=0.912 \\
=0.179 \\
=1.042 \\
=2.031\end{array}$ & ICV (Poland) & $=6.630$ \\
\hline
\end{tabular}

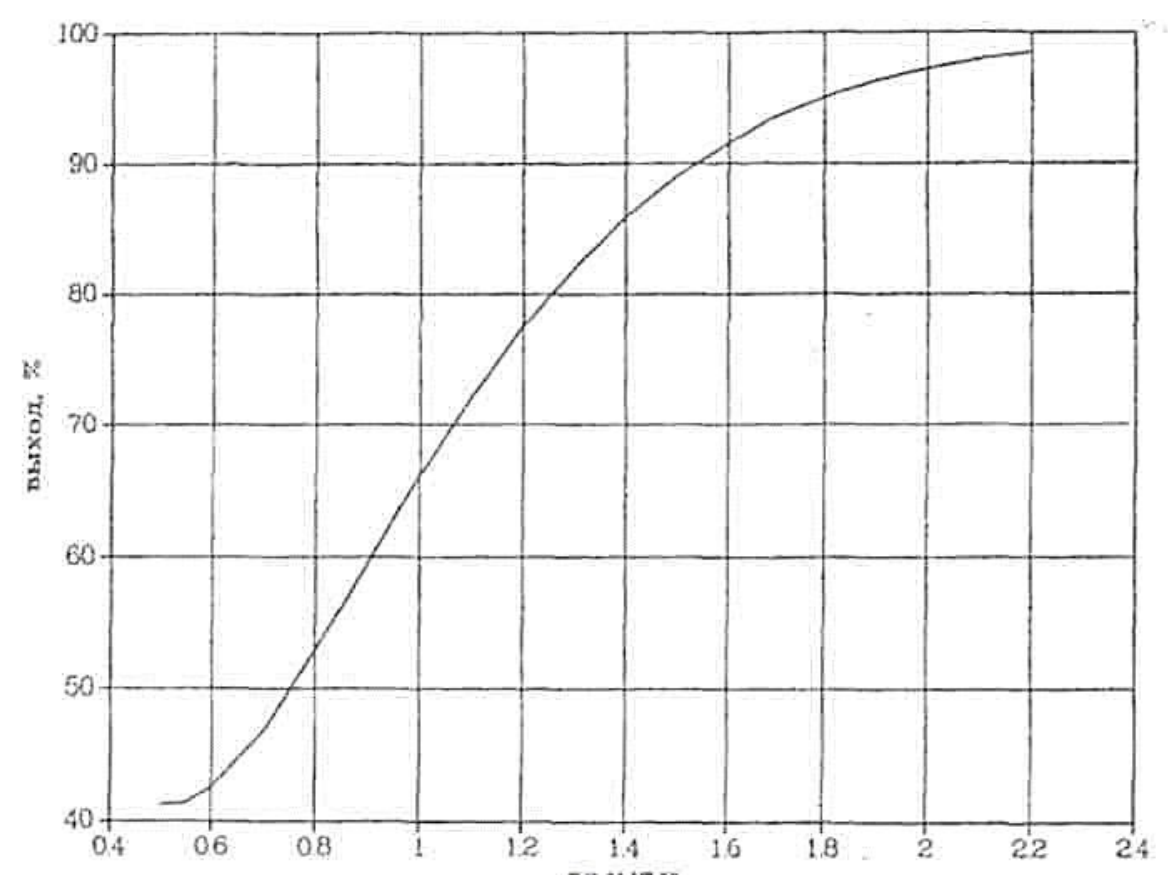

Рисунок 2 - Зависимость выхода необходимой фракции от времени протекания процесса измельчения стекла на струйной мельнице.

Таблица 2

\begin{tabular}{|c|c|c|c|c|c|c|c|c|c|c|c|}
\hline $\mathrm{T}, \mathrm{ч}$ & 0,3 & 0,5 & 0,7 & 0,9 & 1 & 1,1 & 1,3 & 1,5 & 1,7 & 1,9 & 2 \\
\hline $\mathrm{У}, \%$ & 82,08 & 41,2 & 46,89 & 59,54 & 66,09 & 72,15 & 82,08 & 89,005 & 93,49 & 96,25 & 97,18 \\
\hline
\end{tabular}

Вид полученной зависимости представлен на рисунке 2. Неадекватные модели поведения кривой при $\mathrm{t}<0,5$ ч объясняются тем, что затраты энергии на пластические деформации становятся преобладающими, когда основная масса частиц достигает достаточно малого размера. До этого момента все приведенные выше рассуждения не имеют места

$\mathrm{y}=1-16,3173 \mathrm{t}^{2 * \mathrm{e}-3,8739}$.

Итак, перед нами регрессионная модель (6) зависимости выхода стекла крупностью - 0,2 + 0,1 протекания процесса измельчения на струйной мельнице удовлетворяющая всем необходимым условиям, при $0,5<\mathrm{t}<\infty$ функция монотонно возрастает и при $\mathrm{t} \rightarrow \infty \mathrm{y} \rightarrow 1$. При проведении расчетов с базами данных Quattro Pro. C помощью разработанной математической модели по оптимизации измельчения стекла в струйной мельнице решена задача, связанная с максимальным выходом стекла необходимого класса крупности при минимальных энергетических затратах до времени.

Итак, перед нами регрессионная модель (6) зависимости выхода стекла крупностью - 0,2 + 0,1 протекания процесса измельчения на

ISPC Modern research and development, Philadelphia, USA

струйной мельнице удовлетворяющая всем необходимым условиям, при $0,5<\mathrm{t}<\infty$ функция монотонно возрастает и при $\mathrm{t}-\infty$ у $\rightarrow 1$. При проведении расчетов с базами данных Quattro Pro. C помощью разработанной математической модели по оптимизации измельчения стекла в струйной мельнице решена задача, связанная с максимальным выходом стекла необходимого класса крупности при минимальных энергетических затратах до времени. В результате выполненных исследований разработано стекло нового химического состава: $\mathrm{SiO}_{2}-36,5 \%, \mathrm{Al}_{2} \mathrm{O}_{3}-0,25 \%, \mathrm{~F}_{2} \mathrm{O}_{3}-0,005 \%, \mathrm{CaO}-3$, $\%, \mathrm{MgO}-0,99 \%, \mathrm{Na}_{2} \mathrm{O}-10,9 \%, \mathrm{~B}_{2} \mathrm{O}_{3}-14,55 \%, \mathrm{~K}_{2} \mathrm{O}$ - 20,.6\%, $\mathrm{BaO}-12,82 \%, \mathrm{SO}_{3}$ - 0,25\%, которое за счет нейтрализации оксида кремния, обладает пониженной температурой размягчения. Разработана математическая модель по оптимизации процесса измельчения стекла в струйной мельнице, которая объясняет, что затраты энергии на пластические деформации становятся преобладающими, когда основная масса частиц достигает достаточно малого размера. Найден максимальный выход класса крупности $-0,2+0,1$, равный $89,9 \%$, за 


\begin{tabular}{|c|c|c|c|c|c|c|}
\hline Impact Factor: & $\begin{array}{l}\text { ISRA (India) } \\
\text { ISI (Dubai, UAF } \\
\text { GIF (Australia) } \\
\text { JIF }\end{array}$ & $\begin{array}{l}=1.344 \\
=\mathbf{0 . 8 2 9} \\
=\mathbf{0 . 3 5 6} \\
=1.500\end{array}$ & $\begin{array}{l}\text { SIS (USA) } \\
\text { PИHЦ (Russia) } \\
\text { ESJI (KZ) } \\
\text { SJIF (Morocco) }\end{array}$ & $\begin{array}{l}=0.912 \\
=0.179 \\
=1.042 \\
=2.031\end{array}$ & ICV (Poland) & $=6.630$ \\
\hline
\end{tabular}

минимальное время измельчения, которое составляет 1,5 часа работы мельницы. При этом удельная поверхность частиц приближается к удельной поверхности предельно измельченного стеклянного порошка.

\section{References:}

1. Razumov KA (1982) Proektirovanie obogatitel'nyh fabrik. -L.Nedra, 1982. -pp.517.

2. Ivanova VP, Kasatov EK, Krasavina TN, Rozanov EL (1974) Termicheskij analiz mineralov i gornyh porod. - L.: Nedra, 1974.pp.69.

3. Azarov L, Burger M (1961) Metod poroshka v rentgenografii. - M. : IL, 1961. - pp.363.

4. Hejker DM, Bevin LM (1963) Rentgenovskaja difraktometrija. -M. : Fizmat., 1963.- pp.380.
5. Pljusina II (1967) Infrakrasnye spektry silikatov. - M.: Izd-vo Moskovskogo universiteta, 1967.- pp.118.

6. Zisman AD (1974) Adgezija zhidkosti i smachivanija.- M. : Himija, 1974. - pp.413.

7. Sobolev V (1949) Vvedenie v mineralogiju silikatov.- L.: Izdatel'stvo L'vovskogo gosudarstvennogo universiteta, 1949. - pp.329. 